\title{
Exploring Noise Sources Using Simultaneous Acoustic Measurements and Real-Time Flow Visualizations in Jets
}

\author{
James Hileman, ${ }^{*}$ Brian Thurow, ${ }^{*}$ and Mo Samimy ${ }^{\dagger}$ \\ The Ohio State University, Columbus, Ohio 43210
}

\begin{abstract}
Work was carried out as part of an ongoing effort to relate instantaneous large-amplitude features of the farfield acoustic to the dynamic evolution and interaction of large-scale structures within the mixing layer of ideally expanded, high-speed, high-Reynolds-number jets. It is believed that such information is essential for a better understanding of jet noise sources and jet aeroacoustic modeling and control. The acoustic measurements were taken with a four-microphone inline array placed $30 \mathrm{deg}$ to the jet axis. Conditional sampling of the data from the microphones was used to create characteristic waveforms for the large-amplitude, far-field sound pressure peaks. The frequency content of these phase-averaged waveforms compare very well with those of the overall acoustic far field at $30 \mathrm{deg}$. A vast majority of the large-amplitude sound events originated between four and nine jet exit diameters downstream of the nozzle. The acoustic measurements were taken simultaneously with real-time flow visualizations to determine the mechanisms that were responsible for the creation of individual far-field acoustic peaks, and these results have identified three noise-generation mechanisms: intense cross-mixing-layer interaction, the tearing of large turbulent structures, and the rollup of large turbulent structures. The simultaneous data also showed that large structures entrain more ambient air into the jet and the mixing layer extends farther into the jet core during intense noise production than during periods of relative quiet.
\end{abstract}

\begin{aligned} & \multicolumn{1}{c}{ Nomenclature } \\ &$a \quad=$ wavelet scale \\ &$b \quad=$ wavelet translation \\ &$c \quad=$ speed of sound \\ &$D \quad=$ jet exit diameter \\ &$S r_{D}=$ Strouhal number based on jet exit diameter \\ &$s \quad=$ radial distance from the jet centerline \\ & to the microphone array \\ &$u_{c}=$ convective velocity of large structures within \\ & the jet mixing layer \\ &$x_{0}=$ downstream distance of the front microphone \\ & within array \\ &$\delta \quad=$ space between microphones within the array \\ &$\sigma \quad=$ standard deviation of the sound pressure data \end{aligned}

\section{Introduction}

$\mathbf{O}$ VER the past 50 years, considerable effort has been made toward reducing the noise created by jet engine exhaust. In jets with subsonic convective speeds (average speed of large-turbulence structures within the jet), this component of engine noise is known as turbulent mixing noise because it is due to the mixing of the exhausting high-speed jet with the surrounding ambient air. In spite of much effort, the mechanisms that are responsible for the generation of turbulent mixing noise are still not well understood. Many of the average properties of turbulent mixing noise are well known, but not the underlying mechanisms responsible for its creation. The goal of this research effort is to gain a better understanding of the intimate relationship between turbulence and mixing noise in an ideally expanded, high-speed jet.

Turbulent mixing noise is highly directional within the acoustic far field. The noise emission is the most intense at angles close to the downstream jet centerline. The preferred angle has been measured

Received 29 October 2001; revision received 15 July 2002; accepted for publication 19 July 2002. Copyright (C) 2002 by the American Institute of Aeronautics and Astronautics, Inc. All rights reserved. Copies of this paper may be made for personal or internal use, on condition that the copier pay the $\$ 10.00$ per-copy fee to the Copyright Clearance Center, Inc., 222 Rosewood Drive, Danvers, MA 01923; include the code 0001-1452/02 \$10.00 in correspondence with the CCC.

* Graduate Student, Gas Dynamics and Turbulence Laboratory, Department of Mechanical Engineering. Member AIAA.

${ }^{\dagger}$ Professor, Gas Dynamics and Turbulence Laboratory, Department of Mechanical Engineering; samimy.1@osu.edu. Associate Fellow AIAA. to vary from 25 to $45 \mathrm{deg}$ with respect to the downstream jet axis. ${ }^{1}$ An angle of 30 deg was measured with a peak frequency of approximately $3 \mathrm{kHz}\left(S r_{D}=0.2\right)$ for the jet used in this study. ${ }^{2}$ The majority of turbulent mixing noise emanates from a region that includes the end of the potential core. This determinationhas been made by measuring the noise intensity globally over the acoustic near field. ${ }^{1,3,4}$ By the use of the correlation between velocity fluctuations inside the jet and the far-field acoustic pressure, the noise source region of a Mach number 0.98 jet was determined to be between 5 and 10 jet diameters downstream of the jet exit. ${ }^{5}$ By the utilization of various microphone arrays, several research groups have found that the high-frequency noise from high subsonic jets is generated near the nozzle exit, whereas lower-frequency noise originates farther downstream. ${ }^{6-8}$

In studies of low-speed jets and mixing layers, the noise creation process has been linked to vortex pairing. ${ }^{9-11} \mathrm{~A}$ direct numerical simulation of a low-Reynolds-number, Mach 0.9 jet showed the farfield noise originated from a region where the computed Lighthill noise sources were both strong and rapidly changing in time (see Ref. 12). Sarohia and Massier ${ }^{13}$ performed experiments with highspeed schlieren motion pictures that were synchronized with nearfield pressure measurements. Their study of excited subsonic jets with Mach numbers ranging from 0.1 to 0.9 and Reynolds numbers of up to $10^{6}$ found that large instantaneous pressure pulses were formed whenever two large-scale structures merged; however, the passage of a large structure did not significantly change the nearfield pressure signal. These results all indicate that a time-varying source is required to generate noise.

In the first phase of the current research, Hileman and Samimy ${ }^{2}$ used a dual-microphone array located $30 \mathrm{deg}$ with respect to the jet axis for noise source location, as well as simultaneous flow visualization using a double-pulse laser with two charge-coupled device (CCD) cameras, to explore the causes of noise emission within the same high-Reynolds-numberideally expanded Mach 1.3 jet used in this study. The results showed that interactions between large structures across the jet mixing layer and "tearing" of large-scale structures are two likely mechanisms of intense noise production. Obviously, both of these events would cause a rapid temporal change in a large-scale turbulent structure. This is consistent with the previously mentioned studies.

The convective velocity of large-scale structures within the jet mixing layer is a particularly important parameter in this study because it is used to track the location of noise producing events before and after noise is generated. Theoretical equations developed by 
Bogdanoff ${ }^{14}$ and Papamoschou and Roshko ${ }^{15}$ predict a convective velocity of $206 \mathrm{~m} / \mathrm{s}$ and a convective Mach number of 0.6 for the jet in this study. The actual convective velocity of the large-scale structures has been found to deviate substantially from the theoretical value. ${ }^{16}$ Detailed experimental results of Thurow et al. ${ }^{17}$ which were obtained in the same jet facility as the current work with the use of a megahertz rate flow visualization system, indicate that the convective velocity is much higher than the theoretical value. With a large data set of 320 measurements, they found a broad probability density distribution of convective velocities with a mean of $270 \mathrm{~m} / \mathrm{s}$. Based on these findings, a convective velocity of $270 \mathrm{~m} / \mathrm{s}$ is used in the current study.

The examination of turbulent mixing noise in this study focuses on establishing a correlation between the large coherent structures within the jet mixing layer and the measured noise in the acoustic far field. This is commonly known as establishing a causality correlation. In an unconventionalmanner, the acoustic data are examined in the time domain and are only taken to other domains as necessary, when comparison between individual dynamic features and the overall properties of the jet is desired. The jet in this study has a Mach number of 1.3, a nozzle exit diameter $D$ of $25.4 \mathrm{~mm}$ (1 in.) (a dimension that will be extensively used in this work as a reference length scale), a Reynolds number based on the jet diameter of $1.08 \times 10^{6}$, and a potential core length of about six jet diameters. ${ }^{2}$ The region around the potential core, which is where the majority of the far-field acoustic radiation apparently originates, will be the area of primary interest in this work.

\section{Experimental Arrangement}

All of the experiments were conducted in the optically accessed anechoic chamber of the Gas Dynamics and Turbulence Laboratory of The Ohio State University. The facility allows for simultaneous acoustic measurements with real-time flow visualization. This section describes the pertinent components of the experiment, the noise source localization routine, and its validation using a known noise source.

\section{Anechoic Chamber and Jet Facility}

The anechoic chamber facility that was used for these experiments is unique in it allows for the measurement of the jet flow with laser diagnostics in a fully anechoic environment. Thus, simultaneous optical measurements of the jet flow and its acoustic field were conducted. The inner dimensions of the chamber, from wedge tip to wedge tip, are $3.12 \mathrm{~m}$ in width and length and $2.69 \mathrm{~m}$ in height. The chamber was tested for compliance to American National Standards Institue (ANSI) Standard S12.3535, and the results from the tests were within the required tolerance over most of the distances along the microphone paths. ${ }^{18}$ The air for the jet was supplied by two fourstage compressors; it was filtered, dried, and stored in two cylindrical tanks with a total capacity of $42.5 \mathrm{~m}^{3}$ at a pressure of $16.5 \mathrm{MPa}$ $\left(1600 \mathrm{ft}^{3}\right.$ at $2500 \mathrm{psi}$ ). A stagnation chamber was used to condition the jet air before exhausting it through a $25.4-\mathrm{mm}$ (1-in.) nozzle with a lip thickness of $2.5 \mathrm{~mm}(0.1 \mathrm{in}$.), where the inner contour was determined by the method of characteristics for uniform flow at the exit. The actual Mach number of the nozzle was measured as 1.28. Additional details of the anechoic chamber and jet flow facility can be found in Refs. 2 and 18.

\section{Details of the Inline Microphone Array}

The microphone array used in this work relies on the novel application of acoustic principles that was developed with the dualmicrophone array described by Hileman and Samimy. ${ }^{2}$ In that work, and this one as well, the goal was to relate the acoustic far field of the jet to the dynamics of coherentstructures in the flow. The large-scale flow structures are widely believed to create sound that radiates in the preferentialdownstream direction, which, for the current jet, was measured as $30 \mathrm{deg}$ with respect to the downstream jet axis. ${ }^{2}$ Therefore, the microphone array was placed in this direction. Although this unconventionalarray location is not optimal for the localization of noise sources, it was considered necessary to capture accurately the sources of the large-amplitude, low-frequency noise traditionally associated with the turbulence mixing generated by large-scale structures.

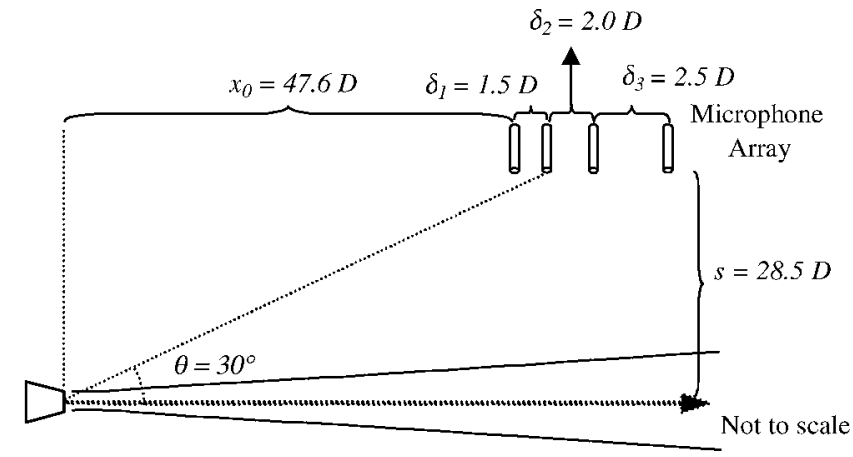

Fig. 1 Schematic of the inline microphone array used to determine noise source location.

The array was composed of four, 6.35-mm ( $\frac{1}{4}$-in.), 4939 Bruel and Kjaer microphones that were separated by $38.1,50.8$, and $63.5 \mathrm{~mm}$ $(1.5,2.0$, and 2.5 in.). These separations, as well as the physical location of the array relative to the jet nozzle, are shown schematically in Fig. 1. An aluminum block surrounded by 5 -cm-thick acoustic foam housed the microphones. Based on the inherent tolerance in the machining of the microphone holding block, the uncertainty in microphone separation was estimated as $0.05 \mathrm{~mm}(0.002 \mathrm{in}$.). All exposed surfaces within the chamber were covered in $1.5-\mathrm{cm}-$ thick acoustic foam. The sound data were acquired with a National Instruments PC-6110E data acquisition board at a rate of 1 million samples per channel per second. This fast acquisition rate was chosen to maximize the accuracy in measuring the time delay between an acoustic signal peak reaching any two of the microphones.

The time delay between an individual acoustic "event" being recorded by two different microphones (phase lag) was combined with knowledge of the array geometry to determine the angle with which the event had approached the array. This angle and the speed of sound in the ambient were then used to determine the locations of the apparent noise sources assuming they were all located on the jet centerline. Further details can be found in Ref. 2. Segments of sound data that were $0.35 \mathrm{~ms}$ in length ( 350 data points) were cross correlated with equal-lengthsegments from the other microphone signals to determine the time delay between the two microphones recording the event. The use of four microphones provided for six different time delays that lead to six different estimates for the event origin. These six locations were then averaged to determine the average noise source location. Any of the six origins that were located outside the range from -5 to $25 \mathrm{D}$, with respect to the nozzle exit, were not included in the average because they were likely in error.

Typical sound pressure data recorded by the microphones that compose the inline array are shown in Fig. 2. The time origin corresponds to the first laser pulse illuminating the flow. The four microphones recorded peaks of similar shape and magnitude, but these peaks are shifted in time, that is, phase shifted. Even at the largest time delay (between the first and last microphones) the overall shape of the sound wave remains substantially unchanged. This manner of determining noise source location is different than those using a cross-spectral method. For that type of technique, which computes an average noise source location and not an instantaneous source, the microphone spacing should not exceed one-half of the wavelength of the sound that is being located. For the technique used here, any microphone spacing can be used, as long as there is a high correlation between the microphone signals for the event of interest.

The large negative sound pressure peak of Fig. 2 recorded around $3.4 \mathrm{~ms}$ was cross correlated for the six different microphone pairs to determine the corresponding time delays and to show how this technique works. Figure 3 shows the $0.35-\mathrm{ms}$ data segments from each microphone that yielded the maximum correlation. Figure 4 shows the same segments shifted by the computed time delays. The time delays between the peaks recorded by microphones 1 and 2 , 1 and 3 , and 1 and 4 were used to shift the signals of microphones 2 , 3 , and 4 to overlap with the microphone 1 signal centered on $3.39 \mathrm{~ms}$. Similarly, the time separation between the signals of microphones 2 and 3 and microphones 2 and 4 were used to overlap their signals to 


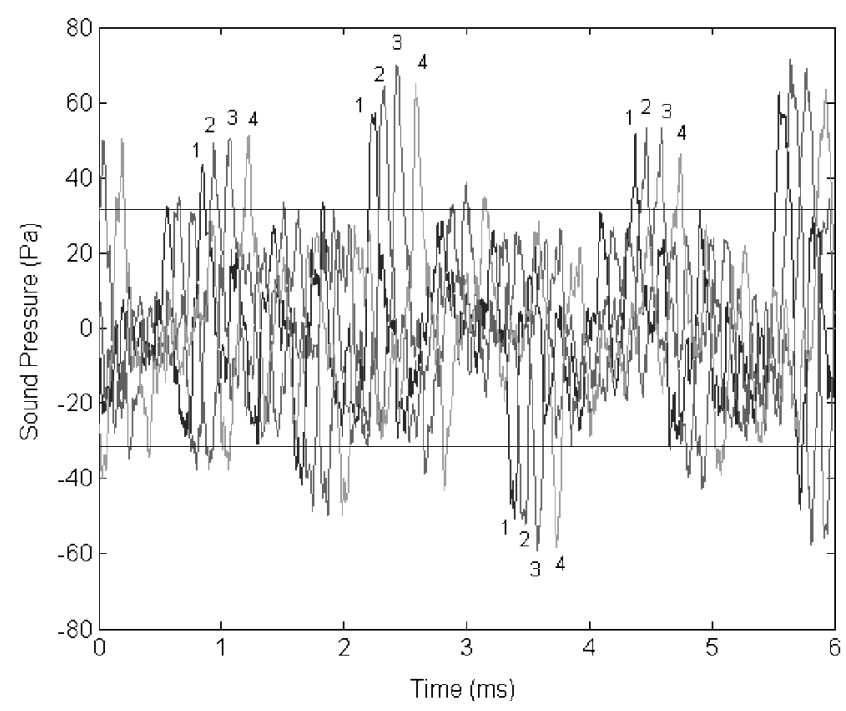

Fig. 2 Typical data recorded by the four microphones of the inline array; time is relative to the first laser pulse illuminating the flow, and the numbers correspond to each of the individual microphone signals.

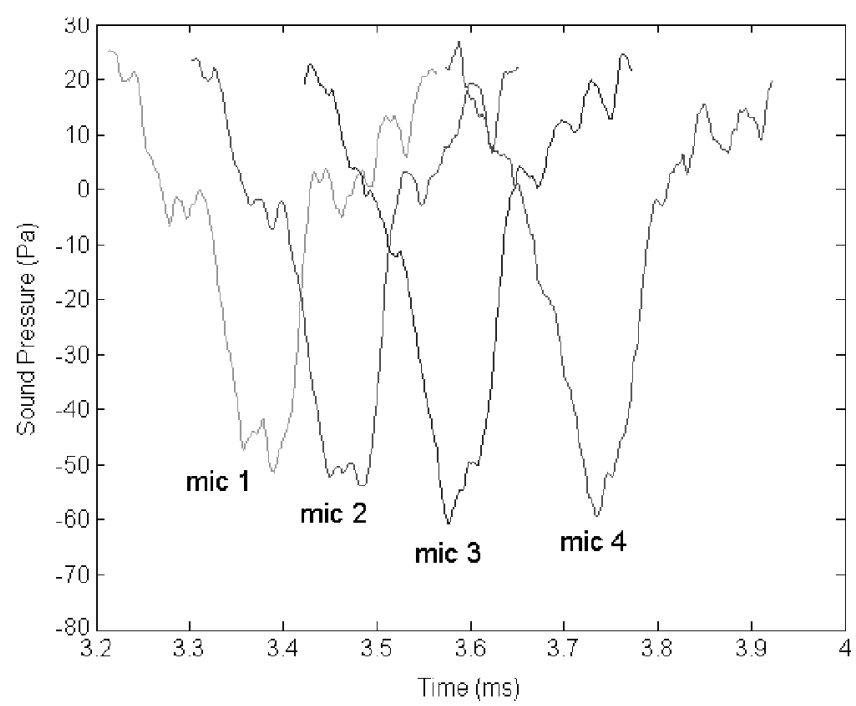

Fig. 3 Time segments for each of the four microphones of the inline array that were used to determine the origin for a large-amplitude peak portion of the time signature in Fig. 2.

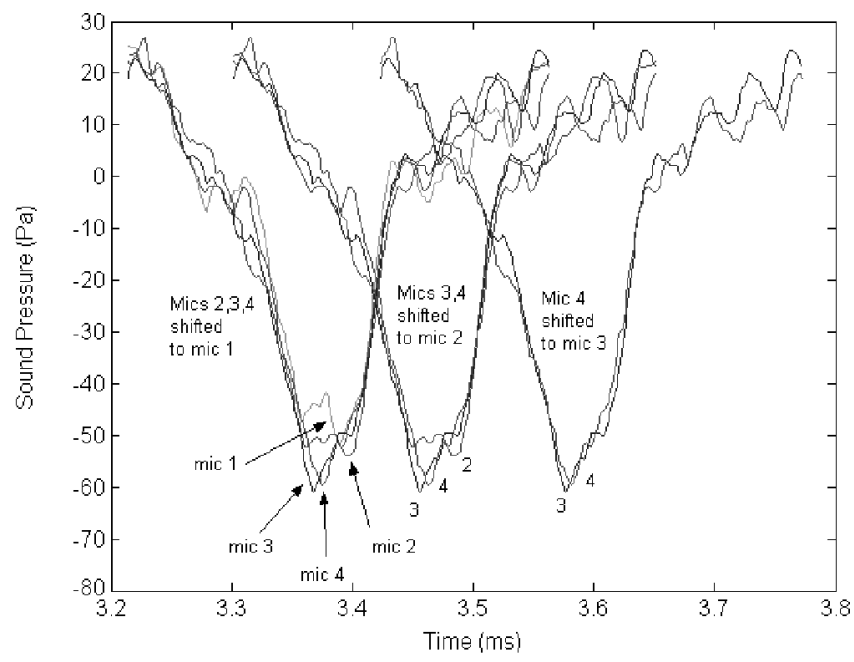

Fig. 4 Time segments of Fig. 3 shifted by the computed maximum correlation times (time separations). that of the microphone 2 signal forming the second group centered on $3.47 \mathrm{~ms}$. Finally, the separation between the peaks for microphones 3 and 4 was used to align the signals of the two microphones centered on $3.60 \mathrm{~ms}$. As seen in Fig. 4, all six time shifts give good visual correlation.

The noise source location technique needs to be instantaneous because the source origins are going to be compared to real-time images of coherent structures and their interaction. To do this, one has to relate the time of noise emission to the time the flow image was captured by laser flow visualization, that is one needs to measure the time lag between the flow visualization and the emission of the sound wave by the noise source. Equations (7-9) of Hileman and Samimy ${ }^{2}$ were used to determine the streamwise location of the noise-emitting region of the jet during the time of laser illumination. Unfortunately, the laser will often not be illuminating the flow while the peak sound generation is occurring; thus, one has to take many sets of data to capture a few noise-producing events while they are creating significant sound. This is clear if one considers that the total illumination time of the flow in the acquired movies is limited to $150 \mu \mathrm{s}$ (approximately 1.6 convectivetime scales of $D / u_{c}$ ), whereas the period of the peak noise at the $30-\mathrm{deg} \operatorname{location}(3 \mathrm{kHz})$ is $333 \mu \mathrm{s}$ (Ref. 2). That is, often the flow is illuminated during a period of intermediate noise generation and not during the most intense noise emission. Ideally, the flow visualizationimage set would span a time period longer than $333 \mu$ s.

\section{Validation of the Inline Microphone Array}

A Hartmann tube fluidic actuator (HTFA) was used to test the ability of this inline array to locate sound sources. ${ }^{19}$ A Hartmann tube consists of an underexpanded jet directed into a coaxial tube, where the open end of the tube is placed within a compression region of the underexpanded jet and the other end of the tube is closed. ${ }^{20}$ The high-speed flow of the jet entering and exiting the tube generates a pulsating flow, as well as an intense tonal sound. An HTFA is created by placing a cylindrical shield between the nozzle and the tube. ${ }^{21}$ This shield covers a large portion of the open area and creates a pulsating jet. The HTFA used in this validation created a pure acoustic tone with a frequency of $3662 \mathrm{~Hz}$ along with five harmonics. The opening of the HTFA was $4 \times 6 \mathrm{~mm}$ and the outer diameter of the HTFA was $12.7 \mathrm{~mm}$. The noise-emittingregion of the HTFA was expected to be within a region close to its exit because this area has been found to be rich in vortical structures. ${ }^{19}$ The HTFA was placed so that the exiting fluid was aligned with the jet centerline, and the back surface of the tube was next to the jet nozzle exit. The inline array was then used to determine the origin of all of the sound events that had amplitude in excess of 1.5 times the standard deviation of the signal. This was performed for $0.6 \mathrm{~s}$ of acoustic data. The predicted downstream locations are given in the probability density distribution of Fig. 5. The peak of the distribution coincides with the exit of the actuator and $90 \%$ of the distribution is within $\pm 0.5 D$ of the actuator exit. Another experiment conducted with a lower pressure HTFA (the frequency content only slightly different from that listed earlier) showed similar results. Because this is a distributed source, rather than a point source, the results indicate that the accuracy of this inline microphone array must be better than $\pm 0.5 D$.

\section{Flow Visualization (Pulse-Burst Laser/Ultrafast Camera System)}

The flow was visualized via scattering of laser light by condensed water particles within the jet mixing layer. The warm, moist air of the ambient is entrained into the cold, dry jet air that is exiting the nozzle, thus forming the jet's mixing layer. On mixing, the moisture contained in the ambient air condenses into small particles that scatter laser light and mark the majority of the mixing layer. The light was provided by an in-house built pulse-burst laser, and the scattered light was captured with a camera from Silicon Mountain Design [(SMD), now a subsidiary of Dalsa, Inc]. Both systems are capable of operating at a megahertz rate. The camera was limited to 17 frames, and the flow visualizationimage sets in this work consist of 16 images. Both a $5-\mu \mathrm{s}(200-\mathrm{kHz})$ and a $10-\mu \mathrm{s}(100-\mathrm{kHz})$ interpulse timing were used. Details of this laser/camera system can be found in Ref. 17. 


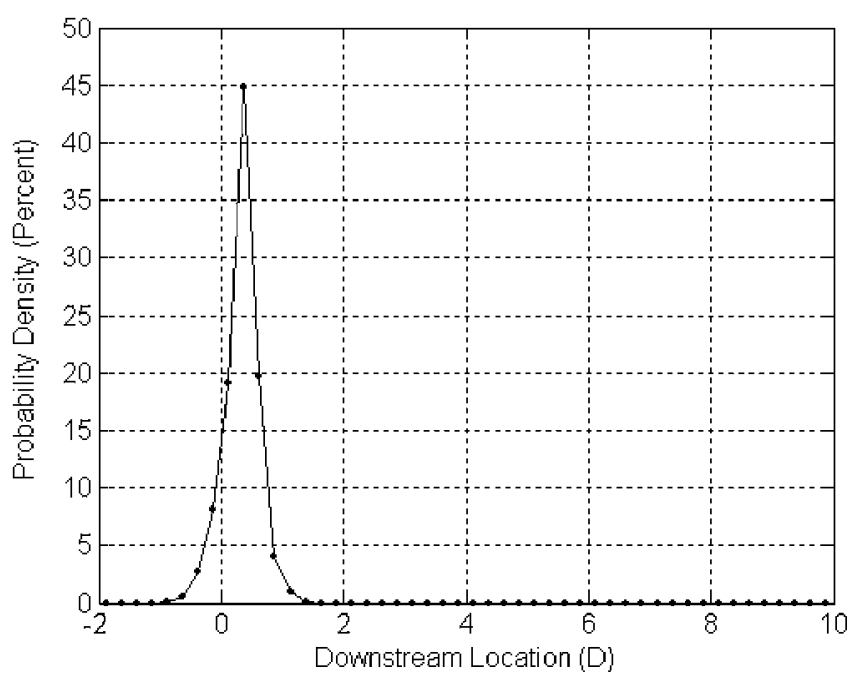

Fig. 5 Probability density distribution of apparent noise source locations for the HTFA used to validate the inline array; the abscissa is relative to the rear of the HTFA, which coincides with the jet exit: •, calculated data points and - , curve fit.

There were 250 sets of simultaneous sound and flow visualization images taken for both of the time separations. The laser was located outside of the anechoic chamber, and its beam was redirected into the chamber through a $2.5-\mathrm{cm}$ opening. A frame structure, which was covered with acoustic foam and connected to the ceiling of the chamber, held the optics that were used to create the laser sheet that passed through the jet centerline along the streamwise direction. The SMD camera was placed inside of the chamber, perpendicular to the laser sheet, capturing the flow over a range of downstream locations from 4.5 to $10.5 \mathrm{D}$. The camera could not be placed outside of the anechoic chamber due to limited laser power and poor sensitivity of the camera. Both the camera stand and the camera were wrapped in acoustic foam to minimize acoustic reflections.

\section{Results and Discussion}

\section{Phase-Averaged Waveform of Large-Amplitude Sound}

\section{Pressure Events}

All of the sound pressure events with acoustic peaks in excess of $2.0 \sigma$ were phase averaged to create a representative waveform. The standard deviation of the sound pressure $\sigma$ was $19 \mathrm{~Pa}$. To obtain the phase-average waveforms, $1 \mathrm{~ms}$ of data before and after every peak exceeding $2.0 \sigma$, where the peaks had been phase aligned, were ensemble averaged. This process was performed separately for the positive peaks (910 events) and negative peaks ( 833 events) to get corresponding phase-averaged waveforms. The analysis was then repeated with a $1.5 \sigma$ threshold. In this case, 2307 positive and 2259 negative peaks were ensemble averaged from $2.0 \mathrm{~s}$ of data to get phase-averaged waveforms. The positive phase-averaged waveforms for both threshold levels are shown in Fig. 6 along with a Mexican hat wavelet, which will be discussed later. All three have been normalized by the standard deviation of the acoustic data. The negative waveforms, not shown here, bear enough similarity to the positive waveforms of Fig. 6 that, if they were rotated 180 deg about the origin, the corresponding positive waveform would be created.

The shapes of the waveforms are quite remarkable. They have sharp, distinct peaks with side lobes on both sides of the central peak. The central peak of the $2.0 \sigma$ threshold waveform has a magnitude of about 2.6, whereas the side lobes have absolute values of about 0.7 and 0.9 . The main peak for the $1.5 \sigma$ threshold waveforms has a magnitude of 2.2, whereas the side lobes have equal values of about 0.6 . Because the $1.5 \sigma$ waveform has equal magnitude side lobes, the asymmetry in the side lobes of the $2.0 \sigma$ waveform must be due in some way to the higher intensity of those sound waves.

The phase-averagedwaveform also shares a strong similarity with the Mexican hat wavelet. A wavelet analysis is often used to determine how the frequency of a signal is changing with time. In this type of analysis, a wavelet is convoluted with the signal of interest. To determine what the frequency content is within the signal,

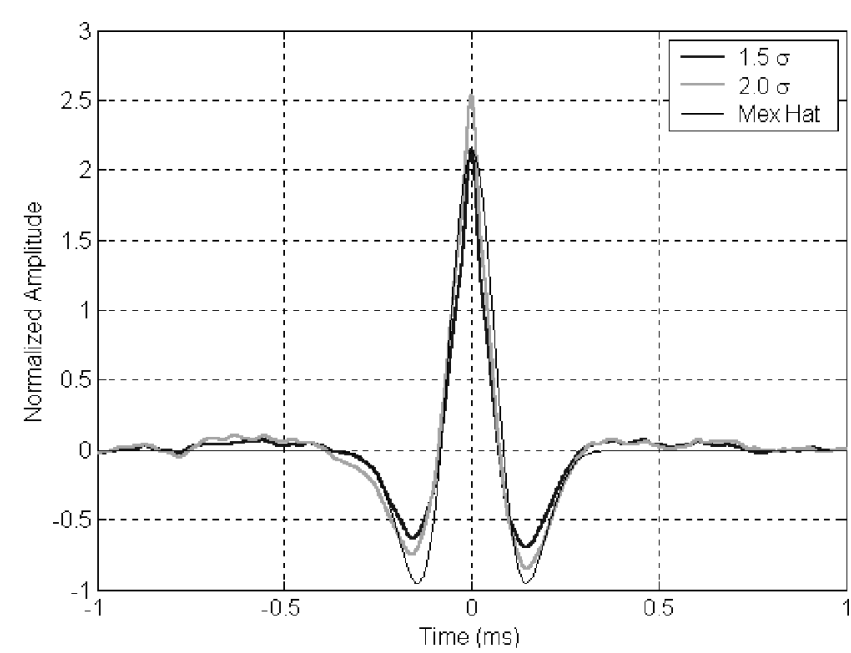

Fig. 6 Phase-averaged, positive, large-amplitude, sound pressure waveforms for all of the time trace segments with a peak amplitude larger than $1.5 \sigma$ and $2.0 \sigma$, along with a Mexican hat wavelet (amplitude normalized by the standard deviation).

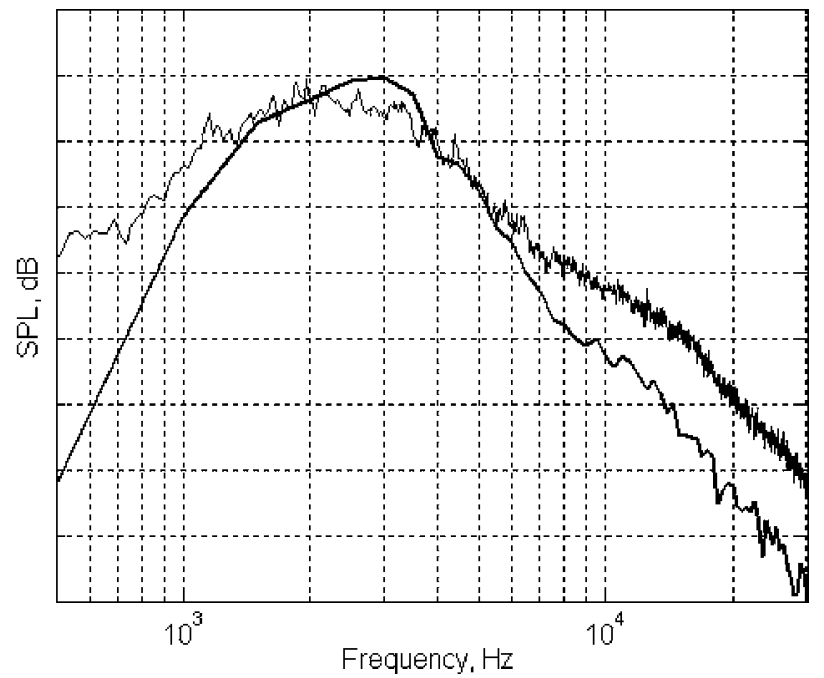

Fig. 7 Overall spectrum and the spectrum for the $1.5 \sigma$ average waveform: —, waveform spectrum.

the wavelet is stretched or compressed by a multiplier known as the scale. The convolution is then performed with the wavelet for varying time delay, which is referred to as translation. When the scale and the translation of the wavelet are changed, the energy contained within various scales (frequencies) can be determined over the duration of the signal. There are an infinite number of wavelets available for study, but a few are commonly used. One of these is the Mexican hat wavelet, which got its name from its similarity to a Mexican sombrero. The Appendix goes into further details of the Mexican hat wavelet and its use in signal analysis. As mentioned previously, Fig. 6 also includes a Mexican hat wavelet that was created via Eq. (A2) of the Appendix with a translation of $0.5 \mathrm{~ms}$, a scale of $84 \mu \mathrm{s}$, and a multiplier of 47 . The Mexican hat wavelet has larger magnitude side lobes than either of the phase-averaged waveforms. Otherwise, the match of the wavelet and waveform is good. Because of these similarities, the acoustic data from the simultaneous measurements were transformed with the Mexican hat wavelet to determine the time-varying frequency content of the sound data.

The $1.5 \sigma$ phase-averaged waveform was transformed to the frequency domain and converted to a power spectral density plot. This spectrum is given in Fig. 7 along with the conventional spectrum. The frequency content of the spectrum around the peak frequency from the $1.5 \sigma$ threshold waveform has a remarkable similarity to the conventional spectrum. The strong match of the spectrafrom the 


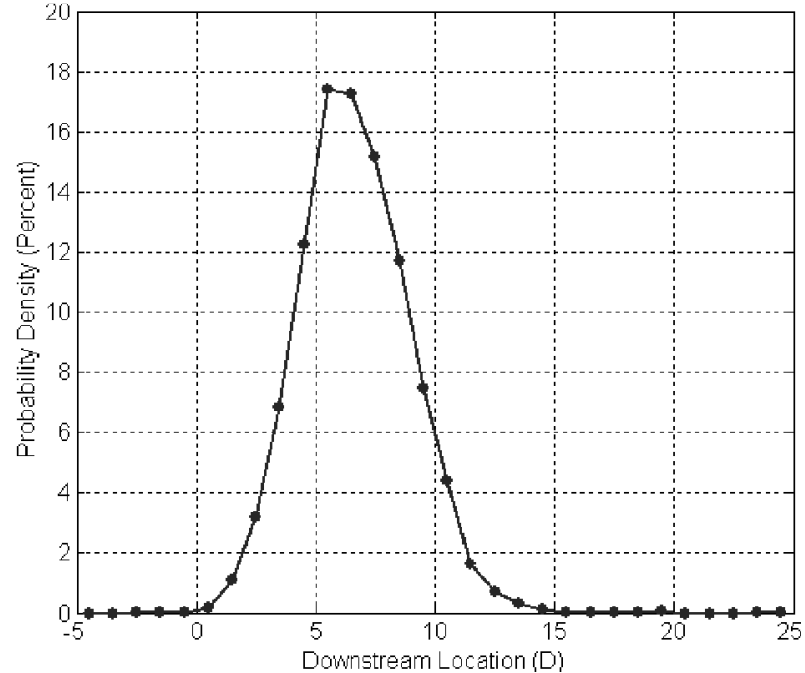

Fig. 8 Probability density of the apparent large-amplitudesound event sources (peak amplitude larger than $1.5 \sigma$ ); $\bullet$, calculated data points that are spaced at $1 D$ and - - curve fit.

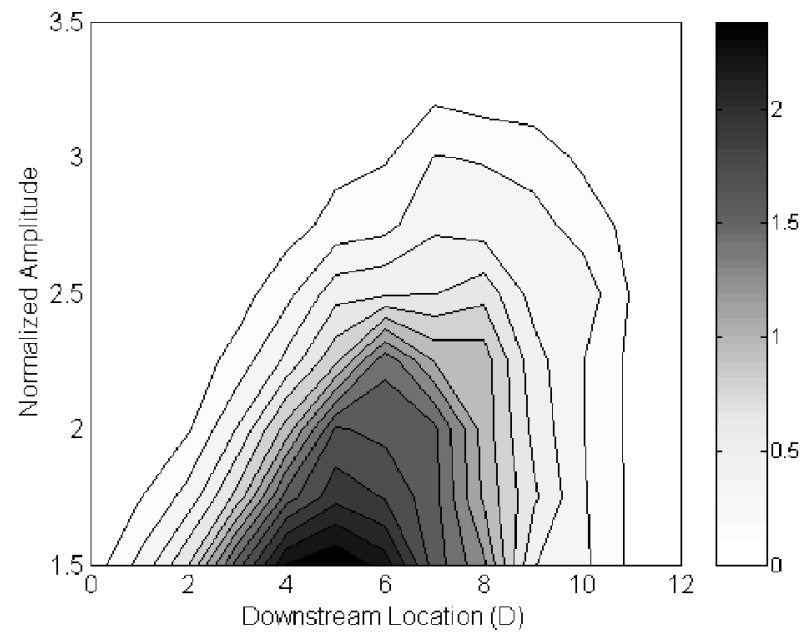

Fig. 9 Two-dimensional probability density distribution showing the downstream locations for sound events of varying amplitude.

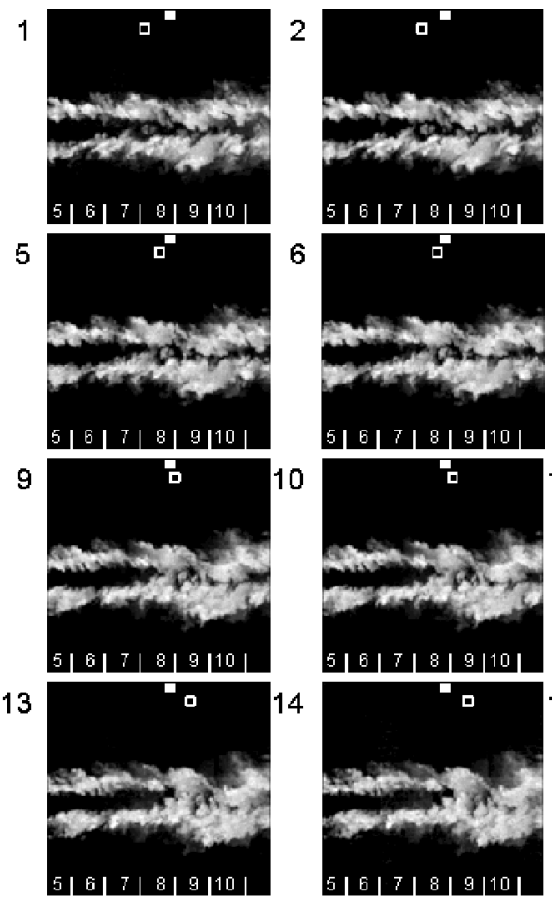

phase-averaged waveforms and the overall far-field radiation shows how well the average waveform is capturing the dominant noise characteristics of the jet at the 30-deg observation angle. This has tremendous implications for understanding the correlation between turbulence structures and far-field acoustic, flow/acoustic control, and aeroacoustic modeling. Because the phase-averaged waveform was created by averaging many individual, large-amplitude sound events, and its spectrum accurately recreates the overall spectrum for the jet, it would appear that the large-amplitude sound events are responsible for the generation of the intense low-frequency sound that is observed at shallow angles to the downstream jet axis.

The phase-averaged waveform bears several similarities to acoustic pressure traces created by the head-on collision of low-Reynoldsnumber vortex rings. Kambe ${ }^{22}$ performed experiments where two vortices were generatedand directed toward each other for a head-on collision. The resulting acoustic pressure time traces were recorded, and the ensemble average of 10 such acoustic time traces was obtained. The resulting waveform has some of the same characteristics as the phase-averaged waveforms given in this work. Both have a large central peak with two side peaks of opposite sign. The side lobes in Kambe's work also have unequal amplitude (as was

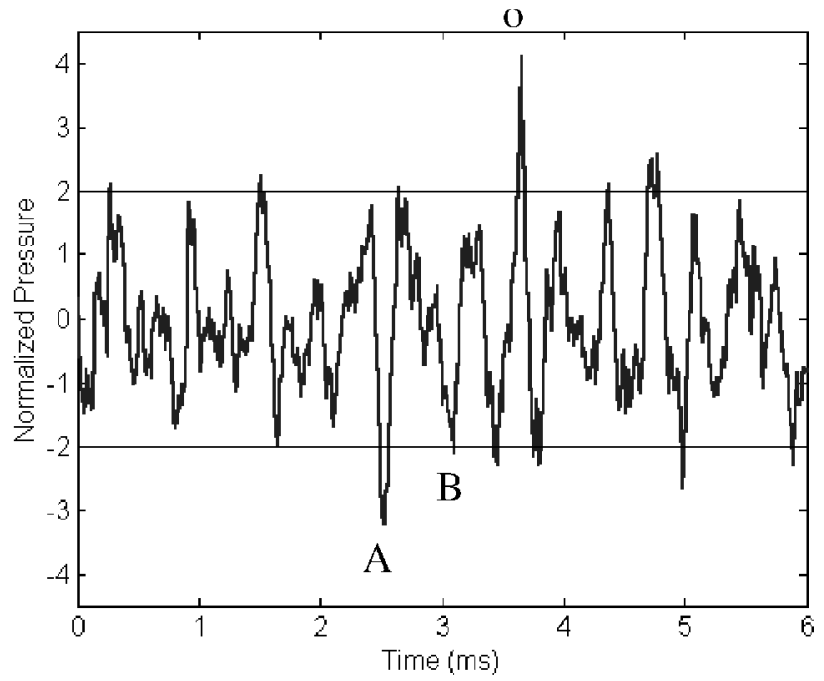

Fig. 11 Far-field acoustic time signature corresponding to flow images of Fig. 10; time zero corresponds to the first laser pulse.

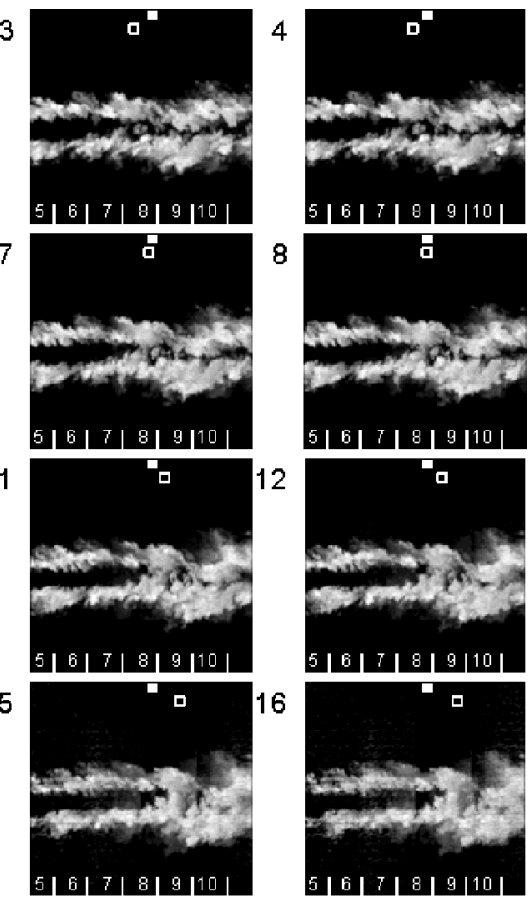

Fig. 10 Example of an image set showing cross-mixing-layer interaction near the origin of an intense sound event. 

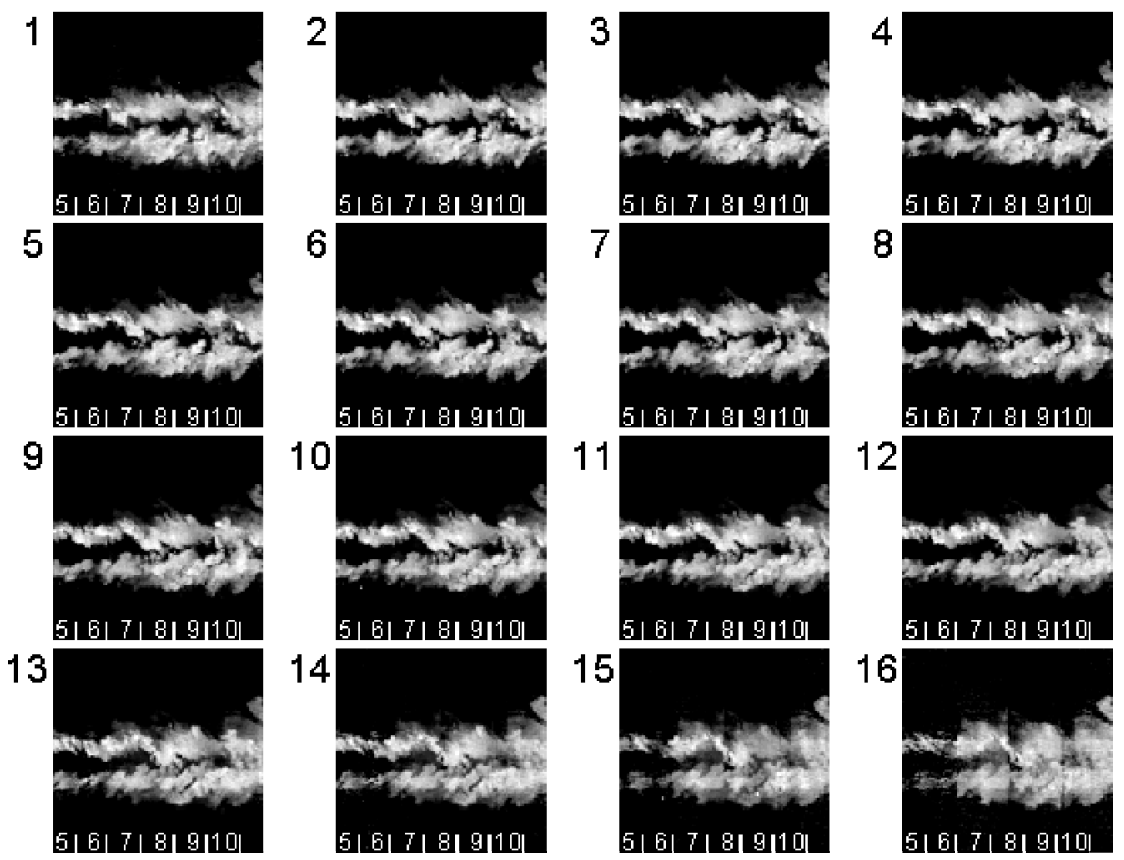

Fig. 12 Example of an image set taken while the microphone array was recording a period of relative quiet.

observed for the $2.0 \sigma$ waveform), and the phase angle (time delay) between side lobes is on the order of that for the phase-averaged waveforms. Inoue et al. ${ }^{23}$ performed a numerical simulation of the head-on collisions of two low-Mach-number vortex rings where the Mach numbers of the two vortices were varied. When the two vortices had equal Mach numbers, the pressure trace did not resemble that of $\mathrm{Kambe}^{22}$ or the phase-averaged waveform of the present work. However, when the ratio of the Mach numbers of the two vortices was two, the shape was very similar to those of the present work. Furthermore, when the ratio was held at two, the pressure trace remained consistent in shape over a range of Mach numbers from 0.15 to 0.3 for the faster vortex. Although there is no direct connection between the noise sources in the Mach 1.3 jet of the present work and the head-on collision of two simple vortex rings, there appear to be similarities in the resulting acoustic waveform between relatively simple vortex ring interactions and the more complex case of turbulent structure interaction in a high-speed jet. This similarity might shed light on how noise is being generated in high-speed jets.

\section{Origin of the Large-Amplitude Sound Pressure Events}

The probability density of the apparent noise emission locations for all of the sound peaks in excess of $1.5 \sigma$ has been plotted in Fig. 8. Data points that had a standard deviation in excess of $2.0 \mathrm{D}$ within the locations determined by the six microphone pairs were not included. This left 5584 data points to be plotted in Fig. 8 for the $2.5 \mathrm{~s}$ of data analyzed. The mean of the noise source locations was $6.7 \mathrm{D}$, and the standard deviation was $2.3 \mathrm{D}$. The vast majority $(74 \%)$ of the noise sources were located in the region between 4 and $9 D$, and $98 \%$ originated between 1 and $12 D$. The lack of noise origins upstream of the jet exit gives further credibility to the accuracy of this noise localization technique.

The absolute magnitude of each sound peak in excess of $1.5 \sigma$ has been plotted vs its origin in the form of a two-dimensional probability density distribution (Fig. 9). Figure 9 was created from the same set of data that was used for Fig. 8. The amplitude has again been normalized by the standard deviation of the acoustic data. The contour levels give the percent of noise events within the two-dimensional bins (the bins are $1 D$ wide along the abscissa and 0.25 units in length along the ordinate). Larger amplitude but less frequently occurring sound events apparently emanate from farther downstream than the weaker, but more frequently occurring, events. For example, one can see from Fig. 9 that events with an amplitude level larger than $2.5 \sigma$ originated downstream of $3 D$. The number of all events drops off considerably after $11 D$.

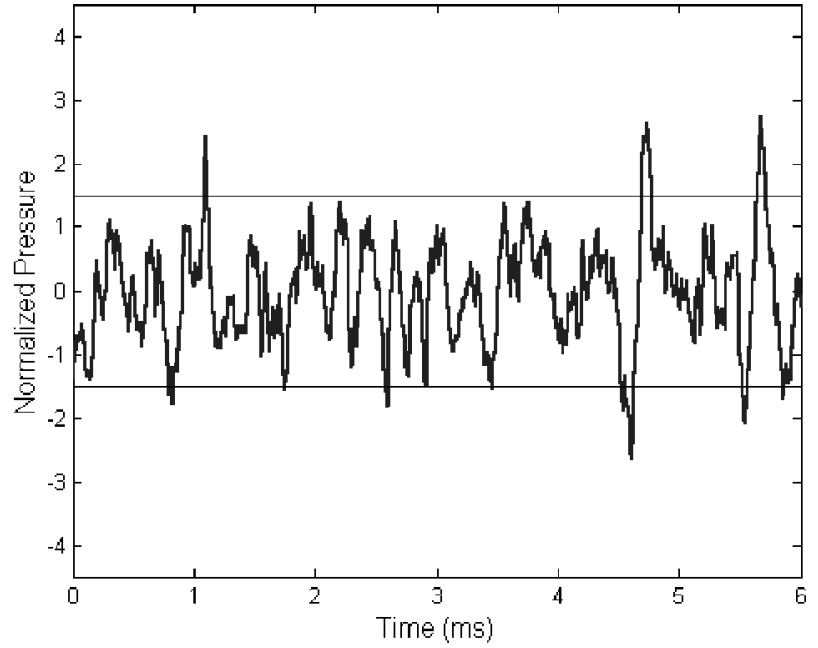

Fig. 13 Far-field acoustic time signature corresponding to the flow images of Fig. 12.

\section{Simultaneous Flow/Acoustic Results}

After the locations for every large-amplitudepeak (using a threshold of $1.5 \sigma$ ) within the 2.5 -s data set were calculated, they were examined to determine which noise generating events would have occurred during the flow visualizations. Any large-amplitude peak that 1) was created during or within a short period of time either before or after the flow visualizations, 2) had an amplitude over $2.0 \sigma$, and 3) had an origin within the field of view was further analyzed. This increased amplitude was deemed appropriate because the phenomena that cause larger amplitude sound waves should be more apparent. These requirements reduced the number of sound pressure events to be analyzed from several thousand to 22 events with $5 \mu$ s between consecutive flow visualization images and 32 events with $10 \mu$ s between flow images.

To understand fully what phenomena are creating these large sound pressure peaks, it is also insightful to examine flow visualization movies that coincide with periods where the microphone array was not recording any large-amplitude events, which is referred to in this work as a "relatively quiet period." A period of relative quiet was defined as one where the array did not record any sound waves in excess of $1.5 \sigma$ over a length of time of at least $0.6 \mathrm{~ms}$. This time span 

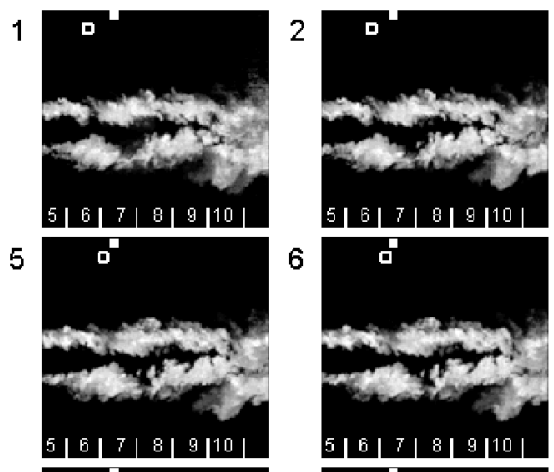

9
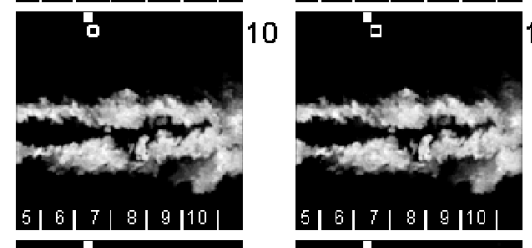

13

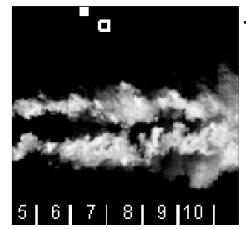

14

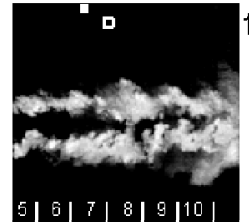

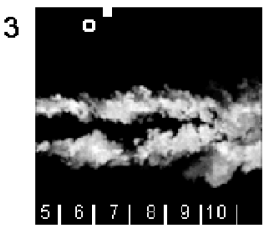

7

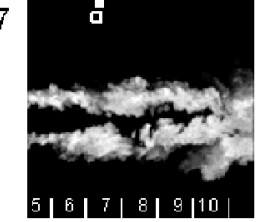

11

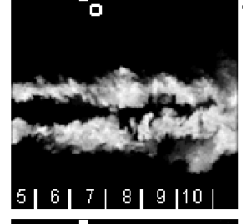

15

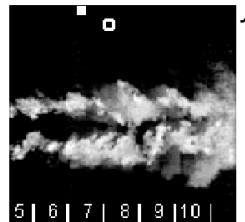

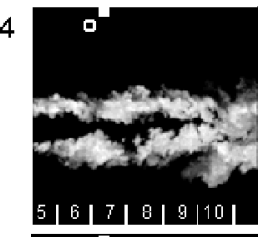

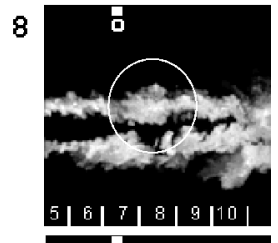

12

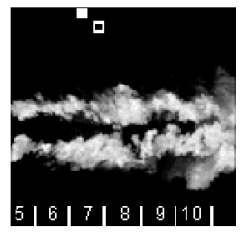

16

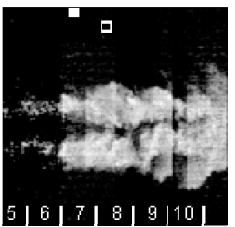

Fig. 14 Example of an image set that shows structure rollup near the origin of an intense sound event; rollup is circled in image 8.

is approximately six convective timescales of $D / u_{c}$. A convective timescale is a time period over which a large-scale structure would travel a distance comparable to the structure's length scale, which is taken to be $D$ here. During such relatively quiet movies, a minimal amount of sound reached the microphone array. Practically speaking, the time traces associated with relatively quiet movies have no large-amplitude peaks between 3.2 and $3.8 \mathrm{~ms}$ after the laser illuminates the flow. There were 12 relatively quiet movies with $10-\mu \mathrm{s}$ spacing, and 18 movies with $5-\mu$ s spacing that met this requirement. Because of the space constraints and because the $10-\mu$ s separation movies show more development of the jet, none of the 5- $\mu$ s movies are presented in this work. However, they were examined in great detail and will be used in the average images that will be discussed in the next section.

The presented data sets consist of images that are separated by $10 \mu$ s and the simultaneously acquired acoustic time signature. Figures 10 and 11 form a typical, simultaneous data set. The images of Fig. 10 can be viewed as a movie of the development of the mixing layer of the jet. In the flow visualization images of Fig. 10 and others to follow, the scattering of laser light via condensed moisture particles created the white, cloudlike areas that mark a major portion of the mixing layer. The flow is from left to right, and the images are numbered. The last few images have poor quality because the laser power dropped significantly toward the end of the set of laser pulses. The apparent streamwise noise emission location (assumed to be on the jet axis) is marked with a closed square. An open square marks the region of the jet that passed through the noise emission location at the time the sound event was created. This region of the jet was assumed to be traveling with a convective velocity of 270 $\mathrm{m} / \mathrm{s}$; hence, the open square is an estimate of the location of the noise source in that frame. The proximity of the squares to the top of the image was chosen to prevent clutter within the images and should not be taken to mean the noise originated above the jet. As was mentioned earlier, the inline acoustic array cannot resolve the cross-stream location of the noise source, and, thus, the noise origin was assumed to be on the jet centerline. The image where both squares are aligned was taken close to, or at, the time of peak noise production. An acoustic time trace from the front microphone of the array is given in Fig. 11. In all of the data shown, the other three microphones recorded phase shifted events similar to that of the front microphone. The time origin on the abscissa corresponds to the first pulse of the laser illuminating the flow.

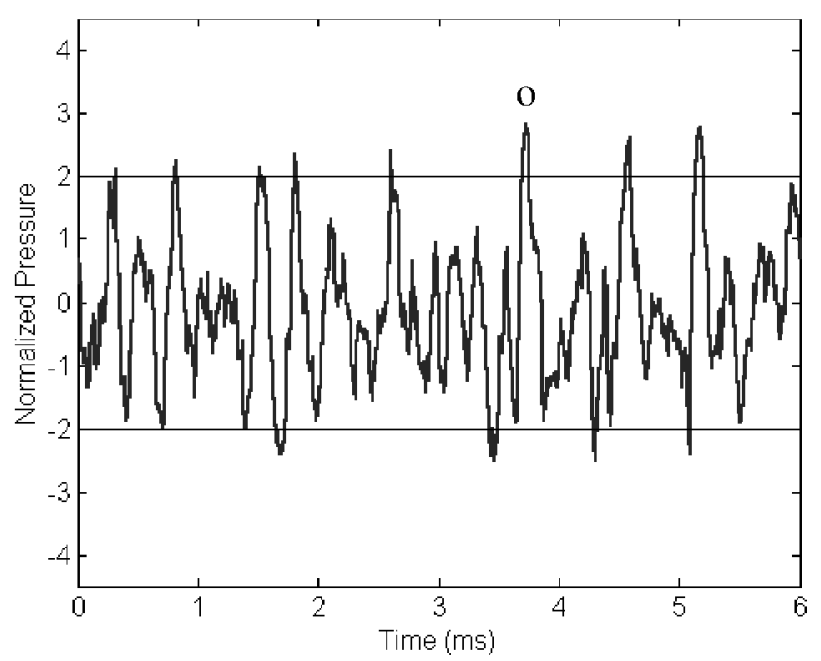

Fig. 15 Far-field acoustic time signature corresponding to the flow images of Fig. 14.

Based on the flow image sets that captured noise generation, three noise-generationmechanisms have been observed. The first is crossmixing-layer interaction, where a large turbulence structure within one side of the mixing layer interacts with another large structure on the opposite side of the mixing layer across the jet core. The second mechanism is the rollup of large structures within one side of the mixing layer. The last mechanism involves a large structure being torn apart. Two of these mechanisms, cross-mixing-layerinteraction and tearing, were observed with dual laser pulse illumination and were presented by Hileman and Samimy. ${ }^{2}$ In this work, examples of all three noise-generation mechanisms are given along with two examples of relative quiet.

Cross-mixing-layerinteraction within the image set of Fig. 10 is the likely cause of the peak that is marked with an "o" in the acoustic time signature of Fig. 11. (The threshold levels of $\pm 2.0 \sigma$ are shown in Fig. 11.) This peak was created at a downstream location of 7.9D, about $70 \mu$ s after the first image was taken. The standard deviation for the noise source location was $0.6 \mathrm{D}$. If the source of the sound moved at a convection speed of $270 \mathrm{~m} / \mathrm{s}$, it would have been at $7.2 \mathrm{D}$ in the first image and at $9.0 \mathrm{D}$ in the last, as is marked with the open 

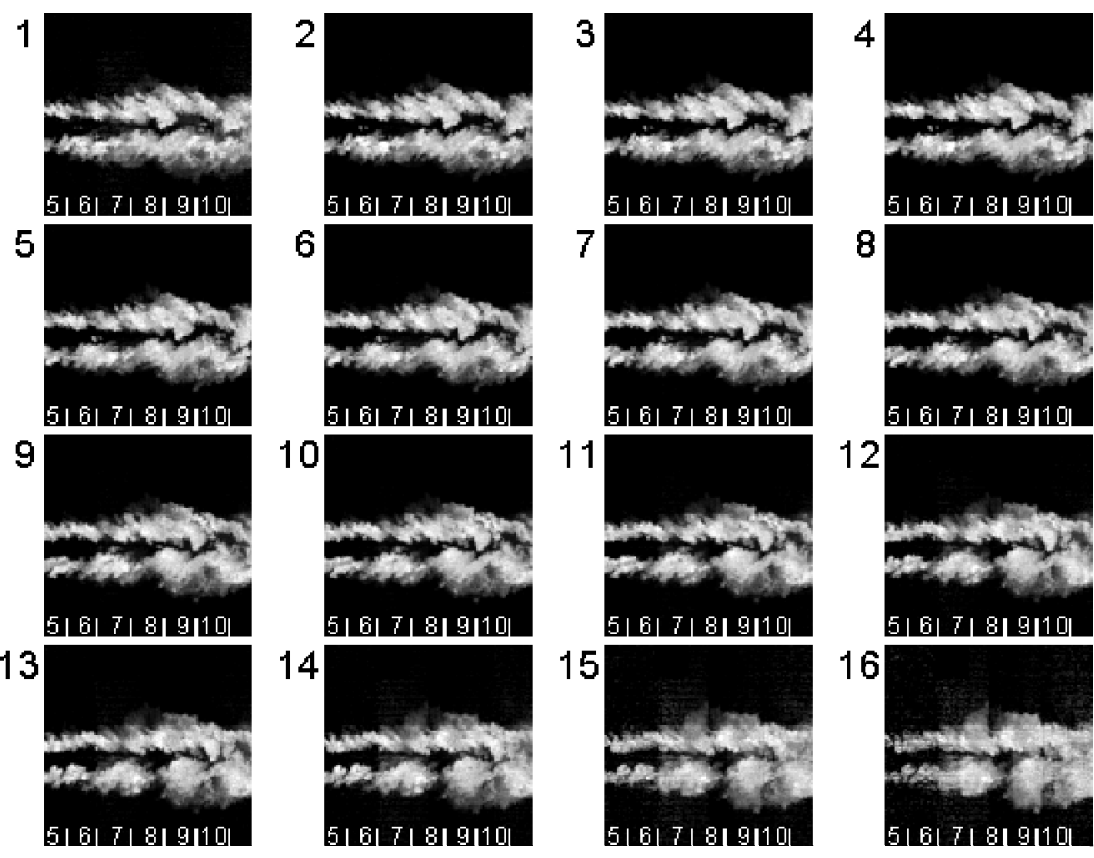

Fig. 16 Example of an image set taken while the microphone array was recording a period of relative quiet.

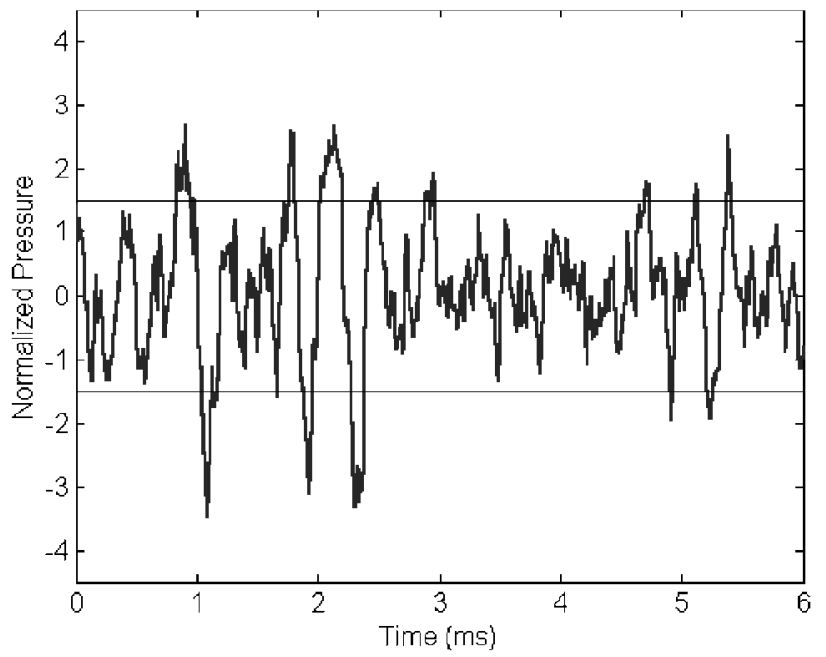

Fig. 17 Far-field acoustic time signature corresponding to the flow images of Fig. 16.

squares. It would appear that the interaction across the two sides of the mixing layer produced the large peak. This interaction region is between 7 and $10 D$ in image number 8 , which was taken at about the same time as the maximum sound generation. There were several other data sets that had similar cross-mixing-layer interaction in areas of noise production.

Two other peaks have been marked in Fig. 10. The one marked A was created at $8.7 D, 1010 \mu$ s before the first image was taken. Thus, the region of the jet that passed through the noise production region, $8.7 D$, at the instant of noise emission would be at $19.4 D$ by the time the first flow image was taken. Similarly, the peak marked $\mathrm{B}$ was created at $5.7 \mathrm{D}, 609 \mu \mathrm{s}$ before the first image. The region of the jet that passed through $5.7 \mathrm{D}$ during noise emission would have convected to $12.2 D$ when the first image was taken. In other words, the regions of the jet, which were responsible for creating sound pressure peaks before $\mathrm{B}$, were downstream of the region being imaged in the flow visualizations. Furthermore, those regions of the jet that caused the peaks recorded after o would be upstream of the imaged flow region.

As mentioned, in addition to the data sets that captured apparent large-amplitude sound emission, there were also data sets that captured the jet while the microphone array was recording a period of relative quiet. One such image set is shown in Fig. 12. As can be seen in the acoustic time trace of Fig. 13, the period of relative quiet is over $1.8 \mathrm{~ms}$ long ( $\sim 18$ convective timescales), and the few peaks in excess of $1.5 \sigma$ were all created long before or after the image set in Fig. 12 was taken. The horizontal lines in the relative quiet acoustic time traces show the $1.5 \sigma$ threshold levels. Based on the lack of large amplitude acoustic events, there should not be any significant noise generating events present in the top portion of the mixing layer. However, in this image set, there is interaction between the two sides of the mixing layer between 7 and $8 D$ in images 9 to 13, but this interaction appears to be much less intense than that of Fig. 10. This subjective interpretation will be validated to some degree in the next section.

The flow visualization image set in Fig. 14 shows the rollup of a large structure within the top half of the mixing layer that appears to have generated significant noise. The structure is located between 7 and $8 D$ within image 8 (the structure is inside the white circle), and the sound event that apparently originated from the same region is marked in the time signature of Fig. 15 with a small o. The apparent origin of the marked peak of Fig. 15 was estimated at $6.5 \mathrm{D}$, and it was created about $70 \mu \mathrm{s}$ after the first (Fig. 14) and $80 \mu \mathrm{s}$ before the last images were taken. The standard deviation in the six individual noise source locations was $1.4 \mathrm{D}$. When a convection velocity of $270 \mathrm{~m} / \mathrm{s}$ is assumed, a large structure at $5.8 D$ in the first frame would be at $6.5 \mathrm{D}$ during noise emission; this same structure (assuming a constant convection velocity) would be located at 7.6D in the last image. The sound wave was created approximately when image 8 was taken. By examination of the rollup process in the flow images, one can see the open square lags the large, developing structure by about one jet diameter. This is expected because the structure is above the jet centerline, and the calculation assumes it to be on the centerline. If the noise origin were $0.5 \mathrm{D}$ above the centerline, then the calculated noise emission location would be $0.8 \mathrm{D}$ farther downstream. Thus, there is very good agreement between the origin of the marked large peak and the large structure rollup. The rollup of large structures was determined to be the mechanism of sound generation in a large number of other image sets.

During the time period when the images in Fig. 16 were taken, the jet was in a period of relative quiet that started at $3 \mathrm{~ms}$ and lasted until $4.5 \mathrm{~ms}$, as is seen in the time signature of Fig. 17. Through analysis of the array acoustic data, it was determined that no noise events in excess of $1.5 \sigma$ were produced during this movie set. The relatively quiet period, using the aforementioneddefinition, is $1.6 \mathrm{~ms}$ long, during which a large-scale structure convecting at $270 \mathrm{~m} / \mathrm{s}$ could travel $16.6 D$. This period of relative quiet is quite remarkable considering the large rollup of successive large structures within 

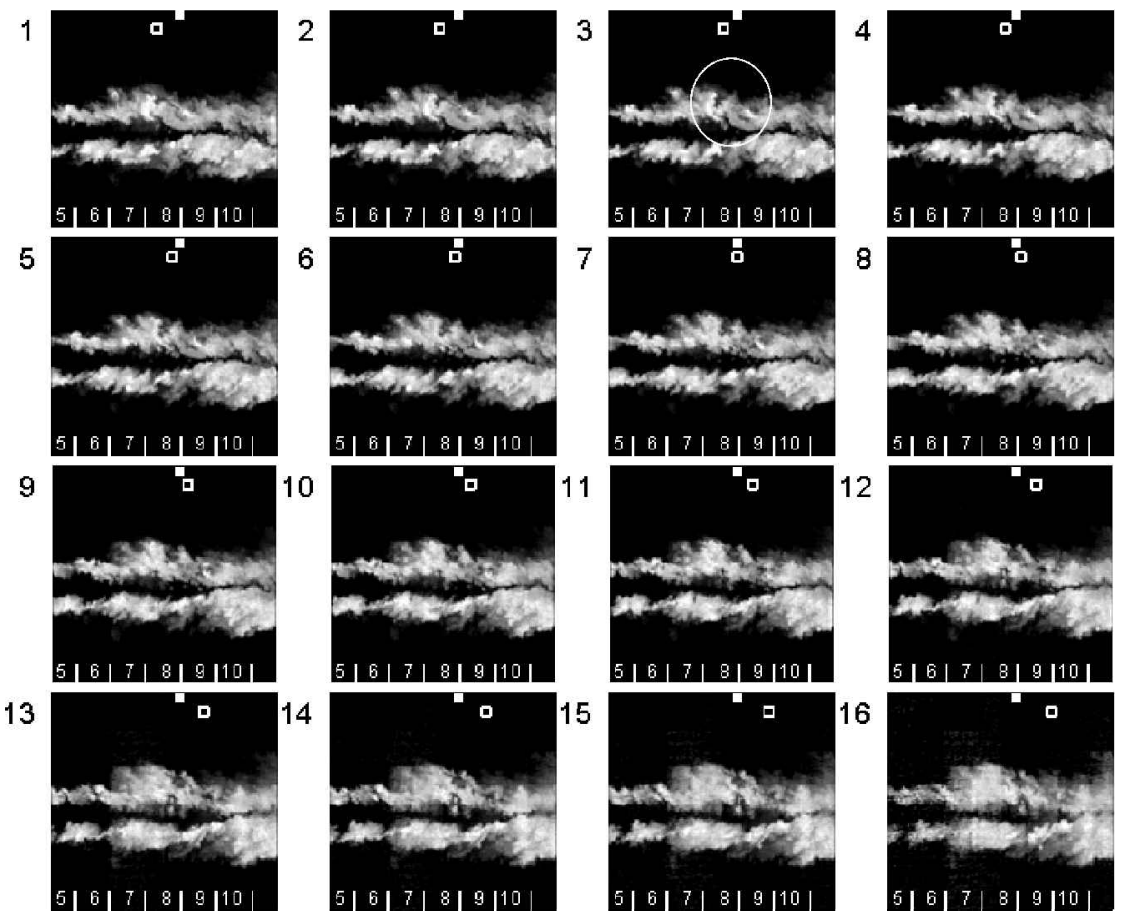

Fig. 18 Example of an image set that shows structure tearing near the origin of an intense sound event; region of tearing is circled in image 3.

the bottom half of the mixing layer (in image 13, the structures are centered at 7 and $9 D$ ). The structure at $9 D$ in image 13 was created by the mixing layer first becoming wavy in appearance (frames 4-6 between 7 and $9 D$ ), and then rolling up into a large, round structure (frames $8-14$ between 8 and $9 D$ ). This process is very much like that described by instability wave theory. ${ }^{1,3}$ The upstream structure (at $7 D$ in image 13) forms between 5 and $7 D$ over the course of the movie set by the merging of several structures.

There were additional cases, not shown here, of large structure rollup within the bottom half of the mixing layer occurring without large-amplitude sound waves reaching the ceiling-mounted microphone array. These vortices, which roll up in either side of the mixing layer, are not axisymmetric. If a source of noise were located on one side of the jet, then it is unlikely that it would radiate uniformly in all azimuthal directions. In addition, the microphoneslocated on the opposite side of the source relative to the jet centerline would receive, if they receive it at all, acoustic radiation that has been refracted (for example, see Ref. 24). Thus, the instantaneous azimuthal radiation pattern caused by a sound source that is located on one side of the jet would probably be nonuniform, and such nonuniformity might explain the inability of this acoustic array to detect the rollup on the other side of the mixing layer as an apparent noise source. Further work is needed to resolve this issue.

The image set of Fig. 18 shows the tearing of a large structure within the top half of the mixing layer that is the apparent cause of the marked peak in the far-field acoustic signature of Fig. 19. This acoustic event has been marked by an o. The apparent origin for the event was at $8.0 D$, and it was created about $60 \mu$ s after the first and $90 \mu$ s before the last images were taken. Hence, the apparent noise source would have been at a location of $7.4 \mathrm{D}$ in the first frame and at $9.2 \mathrm{D}$ in the last (constant convective velocity assumption). The tearing event (marked with a circle in frame 3 ) is occurring over the first four frames of the image set and is distinguishable by the growing separation between structures that are enclosed in the circle. As was the case for the other two noise generation mechanisms, structure tearing was also prevalent in other noise-capturing data sets.

To relate the large-amplitudefar-field acoustic events to the broadband frequency peak, the time-varying frequency of the acoustic signal was computed for each of the cases given earlier by calculating the energy density over time and frequency using the Mexican hat wavelet transformation. The Appendix discusses the Mexican hat wavelet and its associated energy density. The scales that were computed correspond to frequencies between 1 and $10 \mathrm{kHz}$. Based

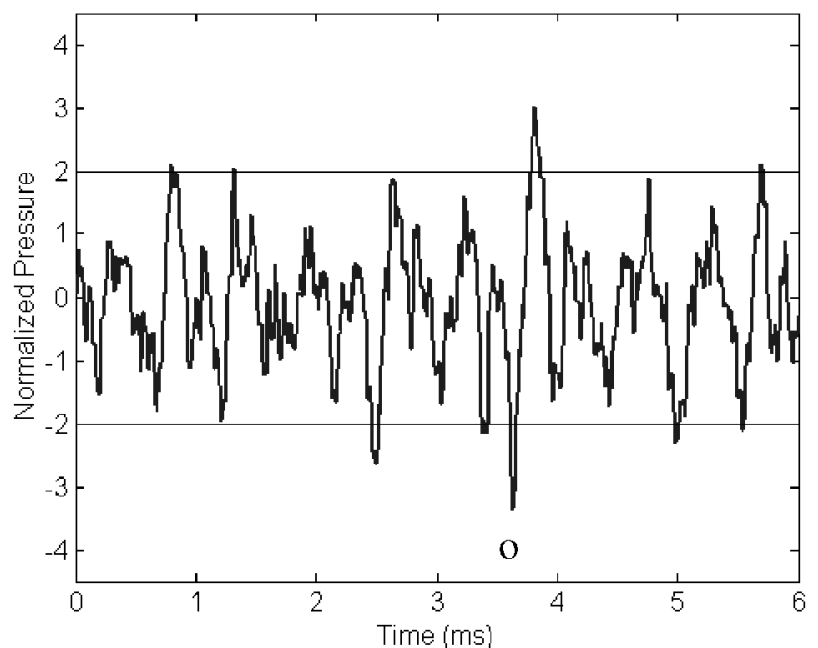

Fig. 19 Far-field acoustic time signature corresponding to the flow images of Fig. 18.

on the energy maps, the large-amplitude peaks (those marked in the preceding acoustic time traces) consistently had large energy content between 2 and $4 \mathrm{kHz}$. The energy density maps for the relative quiet cases had less energy (as compared to the large-amplitude events) within the frequency range between 2 and $4 \mathrm{kHz}$ during the time of relative quiet. Figure 20 shows these two phenomena. It is the energy density distribution for the acoustic time trace of Fig. 17. The large-amplitude events (Fig. 17) all have corresponding high energy levels in the frequency range between 2 and $4 \mathrm{kHz}$ (Fig. 20), whereas the period of relative quiet lacks energy at any frequency. This further supports the argument that these large-amplitudeevents are, in fact, the time domain representation of the broadband peak in the frequency domain that occurs between 2 and $4 \mathrm{kHz}$.

\section{Average Noisy and Relatively Quiet Images}

As mentioned earlier, there were a large number of movies taken during noise production, as well as periods where the microphone array was not recording any large-amplitude sound events. Thus, it made sense to compare the average state of the jet (in terms of the flow images) during these two conditions. The average images of these opposing conditions, as well as the unconditional average 


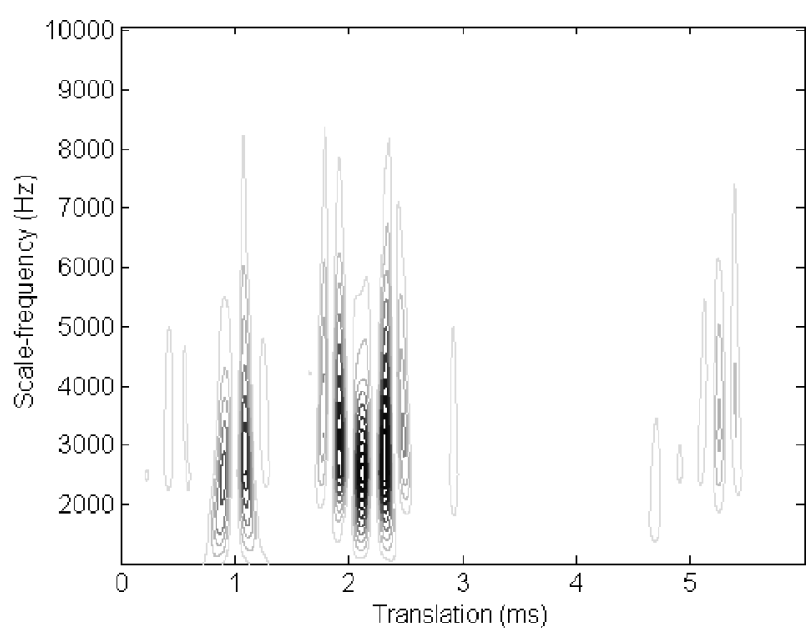

Fig. 20 Energy distribution for the Mexican hat wavelet transformation of the time signature of Fig. 17.

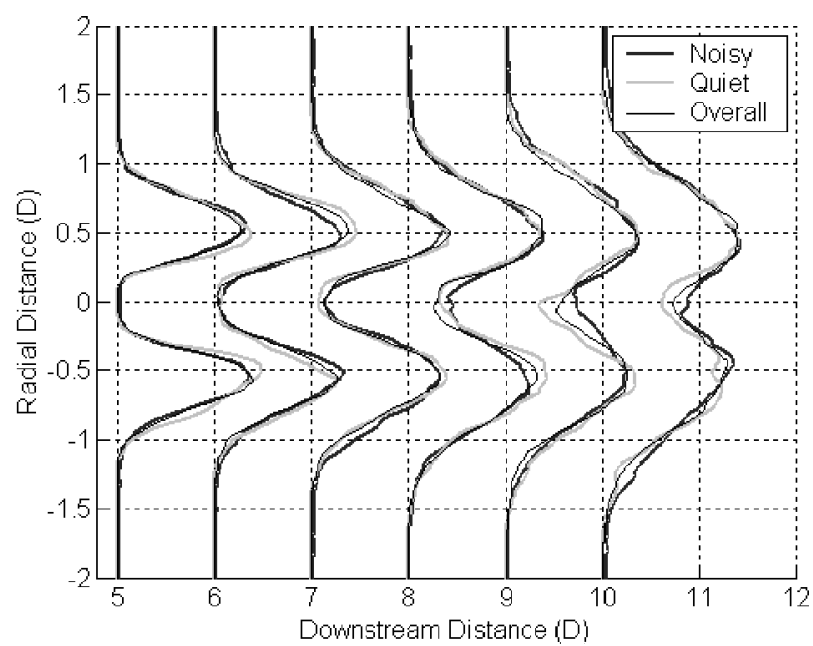

Fig. 21 Plots of the average image intensity for the noise creation (noisy), relatively quiet (quiet), and random (overall) images at various downstream locations.

image of the jet (average of randomly selected images), were obtained to make the comparison.

The random average image of the jet was created by ensemble averaging the seventh flow image, out of the 17 images, from 77 random flow visualization sets. An average flow image over a similar range of downstream locations was given in Ref. 2. Frames from each flow visualization set that had captured a noise event in the process of creating a large sound peak in excess of $2.0 \sigma$ were also ensemble averaged (54 sets in all). Images from each flow visualization image set that had acoustic signatures matching the relative quiet requirements (from the last section, but with a quiet period longer than $0.76 \mathrm{~ms}, \sim 8$ convective timescales) were also ensemble averaged. This set contained 29 images. Before averaging, each of the images was normalized so that its maximum intensity would be one. This was done to eliminate problems caused by variations in shot-to-shot laser pulse intensity.

Figure 21 shows the intensity of each of the three average images (noisy, relative quiet, and random) at select downstream locations. Upstream of $7 D$, the three curves have nearly identical centerline intensity. However, downstream of $8 D$, the noisy image has the largest centerline intensity. Because the intensity is directly related to the amount of condensation particles in the jet, and these particles are a direct result of large structures entraining ambient fluid into the mixing layer, there must be more large structures, or the large-scale structures must be more robust, enabling them to entrain more ambient air into the jet during the period of time the noisy images were taken than during the relative quiet images. This is not surprising because cross-mixing-layer interaction and large-scale structure rollup were considered to be causes of large-amplitude noise production in this work as well as in the work of Hileman and Samimy. ${ }^{2}$ Such an observation is impressive, considering that it was based on an objective sampling of data and that it was not based on a subjective comparison of flow images to noise source locations.

\section{Conclusions}

The focus of this work was on exploring the correlation between turbulence structures and the acoustic far field of a high-Reynoldsnumber, ideally expanded, Mach 1.3 axisymmetric jet. Noise source localization measurements were made with an inline, four-element microphone array located at $30 \mathrm{deg}$, and simultaneous flow visualization was made with a home-built megahertz rate imaging system. The unconventionalarray location of $30 \mathrm{deg}$ was chosen to measure the preferential, high-intensity acoustic radiation in that direction. This flow visualization system, using a pulse-burst laser/ultrafast CCD camera system, was operated at an imaging rate of either 100 or $200 \mathrm{kHz}$.

By phase averaging many large-amplitude sound pressure peaks from the far-field microphones, an average waveform was created. This waveform possesses the same dominant frequency content as the overall acoustic field. This should have tremendous consequences in modeling the acoustic sources. This waveform also resembles a Mexican hat wavelet, and, as such, the Mexican hat wavelet transformation was used to analyze the acoustic signals for their frequency content.

The noise source location was calculated for every sound wave with intensity greater than $1.5 \sigma$ and plotted in the form of a probability distribution. Three-fourths of the sound waves originated between 4 and $9 D$. This is consistent with the results from other researchers, who found the majority of the sound from a high-speed jet originates in the region surrounding the end of the potential core, which, for the jet in this study, was measured between 5 and $6 D$.

Based on the simultaneous flow visualization/acoustic measurements, three apparent noise generating mechanisms were identified within the Mach 1.3 jet. They include cross-mixing-layer interaction, large structure rollup, and structure tearing. Periods of relative quiet (as recorded by the microphone array) were also observed and discussed.

Apparently, asymmetric noise-producing events such as large structure rollups, one of the three identified noise sources, have a pronounced nonhomogenous directivity. For example, when the rollup occurred within the microphone array side of the mixing layer, the array recorded a large-amplitude sound wave, whereas, when it occurred within the other side, the array did not record any significant sound waves.

The average state of the jet during noise emission was compared to that during periods of relative quiet. The average noisy image was obtained by ensemble averaging images taken during time periods of significant noise emission (peak amplitude in excess of $2.0 \sigma$ ), whereas the average relatively quiet image was obtained using the ensemble average of the images taken while the microphone array was recording a period of relative quiet, which was defined as having no sound peaks in excess of $1.5 \sigma$. Based on this comparison, there are more large and coherent structures in the jet that entrain the ambient air into the jet during noise emission than periods of relative quiet. This further supports the idea that large structure rollup and cross-mixing-layerinteraction generate noise.

\section{Appendix: Mexican Hat Wavelet}

The wavelet transformationallows for the analysis of a signal with varying resolution in the time and frequency domains. As is done with a Fourier transformation, the signal is weighted by a function. In the case of the Fourier transformation, the weighting function is $e^{-j 2 \pi f t}$, whereas a wavelet transformation uses a wavelet function for weighting. In both transformations, the weighting is performed via a convolution in time. The general equation for the wavelet transformation, $X_{\text {wav }}(a, b)$, is given as

$$
X_{\text {wav }}(a, b)=\frac{1}{\sqrt{a}} \int_{-\infty}^{\infty} x(t) \Psi\left(\frac{t-b}{a}\right) \mathrm{d} t
$$

where $x(t)$ is the signal that is being transformed, $\psi$ is the mother wavelet being used to analyze the signal, and the $1 / \sqrt{ } a$ quantity 
is used to conserve wavelet energy with wavelet scale. After the transformation, the signal is in a domain of translation $b$ and scale $a$. The translation quantity is very similar to the shifting of the window of the short-term Fourier transform domain. The scale allows for the varying resolution of frequency with varying lengths of time.

The Mexican hat wavelet is a crude, but commonly used, wavelet that is especially well suited to analyze peak locations within a signal because it has a central peak and two small side lobes. The name of the wavelet comes from its resemblance to the cross section of a Mexican sombrero. It is also known as the $g_{2}$ wavelet (second Gaussian wavelet). The Mexican hat wavelet $\psi_{\text {mexh }}$ can be derived by taking the second derivative of the Gaussian probability density function. If normalized for energy conservation purposes, the Mexican hat wavelet is given by

$$
\Psi_{\text {mexh }}(\tau)=\frac{2\left(1-\tau^{2}\right)}{\sqrt{3} \pi^{\frac{1}{4}}} \exp \left(\frac{-\tau^{2}}{2}\right)
$$

where $\tau$ is the modified time given by $(t-b) / a$. Equation (A2) is the form of the Mexican hat wavelet used in this work and shown in Fig. 6.

For this analysis, where the signal was not deterministic, the wavelet equation was not solved analytically. Instead, the signal and Mexican hat function were both discretized. The operation was performed within MATLAB ${ }^{\circledR}$, and the limits of integration were set to the length of the signal. The scale was varied from 25 to $250 \mu \mathrm{s}$, and the translation was scanned over the duration of the acoustic data. The actual equation used for the Mexican hat wavelet transformation is

$$
\begin{aligned}
X_{\text {mexh }}(a, b) & =\frac{1}{\sqrt{a}} \sum_{n=1}^{N} x(n h) \\
& \times \frac{2\left\{1-[(n h-b) / a]^{2}\right\}}{\sqrt{3} \pi^{\frac{1}{4}}} \exp \left\{-[(n h-b) / a]^{2} / 2\right\}
\end{aligned}
$$

where $h$ is the time resolution of the data, equal to $1 \mu \mathrm{s}$. For plotting purposes, the scale was converted to frequency $f$ by the following relationship:

$$
f=(\sqrt{5 / 2}) / 2 \pi a
$$

which is only valid for the Mexican hat wavelet. This relation can be analytically obtained by matching the peak of the wavelet mean spectrum with the peak of the Fourier transform of the cosine wave. In the discussion of the results, $f$ is referred to as scale frequency because it is different from the commonly defined frequency from Fourier analysis.

The energy level at different scales and time can be determined from the wavelet domain, provided that the wavelet does not add energy to the signal. The energy distribution $E(a, b)$ over scale and translation is given by

$$
E(a, b)=(1 / \pi)\left|X_{\operatorname{mexh}}(a, b)\right|^{2}
$$

This equationis similar to that of the power spectraldensity obtained from the Fourier transformation. In the case of the power spectral density, the energy contained in the signal can be obtained by integrating the spectrum over all frequencies. The energy in the signal could also be obtained by integrating the energy distribution over all translations and scales. Typically, sound spectra are converted to a logarithmic quantity (sound pressure level) for analysis; however, the energy distributionmapping in this study was given with a linear scale.

Detailed information on the Mexican hat wavelet and the wavelet transformation can be found in Refs. 25-27.

\section{Acknowledgments}

The support of this research by the Air Force Office of Scientific Research, with John Schmisseur and Steven Walker as the Technical Monitors, is greatly appreciated. The first author would like to thank the Ohio Space Grant Consortium for his doctoral fellowship. The second author is grateful for his National Defense Science and Engineering Graduate fellowship. The authors would like to thank Satish Narayanan of United Technologies Research Center for the help in developing the microphone technique used in this work, as well as stimulating discussions in the field of aeroacoustics. The help of Jeffrey Kastner in researching the wavelet transformation, as well as in the use of the Hartmann tube fluidic actuators, and Marco Debiasi in editing the manuscript, is also greatly appreciated.

\section{References}

${ }^{1}$ Tam, C. K. W., "Jet Noise Generated by Large-Scale Coherent Motion,' Aeroacoustics of Flight Vehicles: Theory and Practice, Vol. 1, edited by H. H. Hubard, Acoustical Society of America, Woodbury, NY, 1991, pp. 311390.

${ }^{2}$ Hileman, J., and Samimy, M., "Turbulence Structures and the Acoustic Far Field of a Mach 1.3 Jet,” AIAA Journal, Vol. 39, No. 9, 2001, pp. 17161727.

${ }^{3}$ Morrison, G. L., and McLaughlin, D. K., "Noise Generation by Instabilities in Low Reynolds Number Supersonic Jets," Journal of Sound and Vibration, Vol. 65, No. 2, 1979, pp. 177-191.

${ }^{4}$ Yu, J. C., and Dosanjh, D. S., "Noise Field of a Supersonic Mach 1.5 Cold Model Jet," Journal of the Acoustical Society of America, Vol. 51, No. 5 Pt. 1, 1972, pp. 1400-1410.

${ }^{5}$ Schaffar, M., "Direct Measurements of the Correlation Between Axial In-Jet Velocity Fluctuations and Far-Field Noise near the Axis of a Cold Jet," Journal of Sound and Vibration, Vol. 64, No. 1, 1979, pp. 73-83.

${ }^{6}$ Simonich, J. C., Narayanan, S., Barber, T. J., and Nishimura, M., "Aeroacoustic Characterization, Noise Reduction, and Dimensional Scaling Effects of High Subsonic Jets," AIAA Journal, Vol. 39, No. 11, 2001, pp. 2062-2069.

${ }^{7}$ Ahuja, K. K., Massey, K. C., and D’Agostino, M. S., "A Simple Technique of Locating Noise Sources of a Jet Under Simulated Forward Motion," AIAA Paper 98-2359, June 1998.

${ }^{8}$ Fisher, M. J., Harper-Bourne, M., and Glegg, S. A. L., "Jet Engine Source Location: The Polar Correlation Technique," Journal of Sound and Vibration, Vol. 51, No. 1, 1977, pp. 23-54

${ }^{9}$ Kibens, V., "Discrete Noise Spectrum Generated by an Acoustically Excited Jet," AIAA Journal, Vol. 18, No. 4, 1980, pp. 434-441.

${ }^{10}$ Colonius, T., Lele, S., and Moin, P., "Sound Generation in a MixingLayer,” Journal of Fluid Mechanics, Vol. 330, Aug. 1997, pp. 375-409.

${ }^{11}$ Mitchell, B., Lele, S., and Moin, P., "Direct Computation of the Sound Generated by Vortex Pairing in an Axisymmetric Jet," Journal of Fluid Me chanics, Vol. 383, Oct. 1998, pp. 113-142.

${ }^{12}$ Freund, J., "Noise Sources in a Low-Reynolds-Number Turbulent Jet at Mach 0.9," Journal of Fluid Mechanics, Vol. 438, 2001, pp. 277-305.

${ }^{13}$ Sarohia, V., and Massier, P. F., "Experimental Results of Large-Scale Structures in Jet Flows and Their Relation to Jet Noise Production," AIAA Paper 77-1350, Oct. 1977.

${ }^{14}$ Bogdanoff, D. W., "Compressibility Effects in Turbulent Shear Layers," AIAA Journal, Vol. 21, No. 6, 1983, pp. 926, 927.

${ }^{15}$ Papamoschou, D., and Roshko, A., "The Compressible Turbulent Mixing-Layer: An Experimental Study," Journal of Fluid Mechanics, Vol. 197, 1988, pp. 453-477.

${ }^{16}$ Murakami, E., and Papamoschou, D., "Eddy Convection in Coaxial Supersonic Jets," AIAA Journal, Vol. 38, No. 4, 2000, pp. 628-635.

${ }^{77}$ Thurow, B., Hileman, J., Samimy, M., and Lempert, W., "Compressibility Effects on the Growth and Development of Large-Scale Structures in an Axisymmetric Jet," AIAA Paper 2002-1062, Jan. 2002.

${ }^{18}$ Kerechanin, C. W., Samimy, M., and Kim, J.-H., "Effects of Nozzle Trailing Edges on Acoustic Field of Supersonic Rectangular Jet," AIAA Journal, Vol. 39, No. 6, 2001, pp. 1065-1070.

${ }^{19}$ Kastner, J., and Samimy, M., "Development and Characterization of Hartmann Tube Fluidic Actuators for High-Speed Flow Control,' AIAA Journal, Vol. 40, No. 10, 2002, pp. 1926-1934.

${ }^{20}$ Hartmann, J., and Trolle, B., "A New Acoustic Generator. The Air-Jet Generator," Journal of Scientific Instruments, Vol. 4, 1927, pp. 101-111.

${ }^{21}$ Raman, G., Kibens, V., Cain, A., and Lepicovsky, J., "Advanced Actuator Concepts for Active Aeroacoustic Control," AIAA Paper 2000-1930, June 2000.

${ }^{22}$ Kambe, T., "Acoustic Emissions by Vortex Motions," Journal of Fluid Mechanics, Vol. 173, 1986, pp. 643-666.

${ }^{23}$ Inoue, O., Hattori, Y., and Sasaki, T., "Sound Generation by Coaxial Collision of Two Vortex Rings," Journal of Fluid Mechanics, Vol. 424, 2000 pp. 327-365.

${ }^{24}$ Freund, J. B., and Fleischman, T. G., "Ray Traces Through Unsteady Jet Turbulence,' International Journal of Aeroacoustics, Vol. 1, No. 1, 2002 , pp. 83-96.

${ }^{25}$ Daubechies, I., Ten Lectures on Wavelets, Society for Industrial and Applied Mathematics, Philadelphia, 1992, pp. 1-106.

${ }^{26}$ Lewalle, J., "Wavelet Analysis of Experimental Data: Some Methods and the Underlying Physics," AIAA Paper 94-2281, June 1994.

${ }^{27}$ Wang, Q., Brasseur, J., Smith, R., and Smits, A., "Application of the Multi-Dimensional Wavelet Transforms to the Analysis of Turbulence Data," International Workshop on Wavelets, Springer-Verlag, New York, 1991. 